\title{
Forschung und Soziale Arbeit zu Queer mit Rassismuserfahrungen
}

Saideh Saadat-Lendle und Zülfukar Çetin

Wir ${ }^{1}$ sind eine queere Gruppe, die sich sowohl akademisch als auch politisch selbstbestimmt gegen Mehrfachdiskriminierungen in der Dominanzgesellschaft $^{2}$ einsetzt.

Es ist uns wichtig, auf dem ersten LSBTI*-Wissenschaftskongress der Bundesstiftung Magnus Hirschfeld (BMH) deutlich $\mathrm{zu}$ machen, dass wir

1 | Wir sind Lesben, Schwule, Trans* und Intergeschlechtliche, Bisexuelle, Frauen und Männer, Tunten und Kampflesben, wir sind dick und hässlich, wir haben Behinderungen, wir sind HIV-positiv und Obdachlose, wir sind Ausländer_innen, wir haben Migrationshintergrund, wir definieren uns als Queer of Color. In unserer langjährigen wissenschaftlichen und zivilgesellschaftlichen Arbeit für Menschen mit Rassismuserfahrungen stoßen wir auf verschiedene Erwartungen von unterschiedlichen Menschen, Gruppen und Institutionen. Manchmal werden wir zu Expert_innen für das Thema Rassismus gemacht, manchmal gelten wir als Repräsentant_innen für Menschen mit sogenanntem Migrationshintergrund, manchmal werden uns unsere Erfahrungen mit Rassismus aberkannt. Manchmal werden wir als Opfer der von Migrant_innen ausgehenden Homophobie und Transphobie betrachtet. Mit unseren Namen, die korrekt auszusprechen so vielen schwerfällt, mit unserem schwer einzuordnenden Äußeren, mit unseren migrantisierten Weiblichkeiten und Männlichkeiten sind wir manchmal Unsicherheitsfaktoren für Kolleg_innen, die mit uns vorsichtig umgehen wollen, weil sie uns nicht diskriminieren wollen. Wir bedanken uns bei Jacek Marjański und Maria Virginia Gonzalez Romero, die gemeinsam mit uns auf dem 1. LSBTI*-Wissenschaftskongress der Bundesstiftung Magnus Hirschfeld ihre Erfahrungen mit Rassismen und Homophobie in ihrer alltäglichen sozialen Arbeit schildern durften.

2 | Das Konzept der Dominanzgesellschaft lehnt sich an das von Birgit Rommelspacher entwickelte Konzept der Dominanzkultur an. Das Konzept der Dominanzkultur geht davon aus, dass sich die Gesellschaft nicht aus einer oder einer begrenzten Anzahl von Perspektiven heraus analysieren lässt, sondern dass unterschiedliche Machtdimensionen die gesellschaftlichen Strukturen und das konkrete Zusammenleben bestimmen, und im Sinne eines Dominanzgeflechts miteinander verwoben sind (vgl. Rommelspacher 2006: 3). 
uns zu der Abkürzung LSBTI* kritisch positionieren. Wir sind der Meinung, dass LSBTI* der sprachlichen Vereinfachung bei der Nennung von Gruppen dient, die mit LSBTI*-Feindlichkeiten konfrontiert sind. Mit der Bezeichnung LSBTI* werden Lesben, Schwule, Bisexuelle, Trans* und Intergeschlechtliche als einzelne Identitäten nebeneinander gestellt und unterschiedliche Diskriminierungserfahrungen auf das Sexuelle bzw. Geschlechtliche reduziert. Dabei werden oft die Menschen vergessen, die gleichzeitig Klassismen und Rassismen ausgesetzt sind.

Ausgehend von dem Motto Identität kennt kein Entweder-Oder (LesMigraS undatiert) befürworten wir den Begriff Queer statt LSBTI*. Mit dem Begriff Queer wollen wir unsere wissenschaftlichen, gesellschaftlichen und politischen Positionen und unser selbstbestimmtes Engagement gegen Mehrfachdiskriminierungen in der weiß-deutschen Dominanzgesellschaft zum Ausdruck bringen. In diesem Beitrag wollen wir als Wissenschaftler_innen, Aktivist_innen, Sozialarbeiter_innen bzw. als Menschen, deren Qualifikationen oft infrage gestellt werden, ein Zeichen gegen institutionelle und alltägliche mehrdimensionale Herrschaftsverhältnisse setzen. Anhand der Analyse ausgewählter Studien zeigen wir auf, wie der von einer Allianz aus Wissenschaft, Zivilgesellschaft und Staat (re-)produzierte Homophobiediskurs Rassismen erzeugt und praktiziert. Dabei gilt es auch, die Repräsentations-, Legitimations- und Gerechtigkeitsansprüche dieser Allianz zu hinterfragen.

\section{Der Repräsentationsanspruch der Zivilgesellschaft}

Sowohl im akademischen als auch zivilgesellschaftlichen Kontext dienen wir oft als Forschungsobjekte: Unsere besondere Lebensweise als Queers mit Migrationshintergrund interessiert die eurozentrische Wissenschaft, Zivilgesellschaft und den Staat. Es werden Studien und Projekte über Themen wie Integration, Migrant_innen, Toleranz und Demokratie, Homophobie, Antisemitismus etc. durchgeführt. Während unsere Weiblichkeiten und Männlichkeiten migrantisiert werden, werden wir mit unseren Homosexualitäten als Teil einer aufgeklärten und demokratischen europäischen Gesellschaft anerkannt. Unsere Familien, Freund_innen, Kolleg_innen mit ihren migrantisierten Heterosexualitäten erklärt man jedoch als einen Gegensatz zu uns.

In der bekannten LSVD-Kampagne »Gül ist lesbisch - Hassan ist schwul - Er/Sie gehören zu uns, Jederzeit«, welche im Jahr 2012 in Berlin ins Leben gerufen wurde, sehen wir z.B. den polarisierenden und inakzeptablen Repräsentationsanspruch des Verbandes: Gül und Hassan werden in der LSVDKampagne als Lesbe und Schwuler mit Homophobieerfahrungen markiert. Doch wie ist das »wir« definiert, zu dem die beiden angeblich gehören? Als größte lesbisch-schwule Organisation der BRD behauptet der Verband, sich 
für die sozialen Rechte der in Deutschland lebenden Lesben, Schwulen und Trans* einzusetzen ${ }^{3}$. Während der von weißen Deutschen geführte LSVD sich u.a. für die Homo-Ehe engagiert, bleiben viele andere Fragen offen - z.B. die nach den Rassismuserfahrungen von Gül und Hassan und den daraus resultierenden Zugangsbarrieren zu Bildung, gut bezahlter Arbeit, zum Wohnungsmarkt oder dem Gesundheitssystem. Die auf dem Plakat Porträtierten werden als Repräsentant_innen der Schwulen und Lesben of Color dargestellt: Sie erscheinen als homosexuelle Opfer eines als homophob imaginierten Islam. Der Kampf des LSVD gegen Homo- und Transphobie wird auf diese Weise zu einem Kampf gegen Muslim_innen.

\section{LEgItIMATIONSANSPRUCh DER WISSENSChAFT}

Während die eurozentrische Wissenschaft kontinuierlich versucht, zu beweisen, dass ein Hassan, der schwul sein soll wie ein $\mathrm{Kai}^{4}$, Opfer seiner Familie, seiner Freund_innen oder seiner Verwandten sei, wird nicht gefragt, ob der Kai, der schwul sein soll wie Hassan, ebenfalls Erfahrungen mit Rassismen macht und Konsequenzen daraus ziehen muss. Die Konstruktion eines schwulen Opfers des Islams wird mit dem Motto »Gül ist lesbisch, sie gehört zu uns, jederzeit!« ausgeweitet. Gül als lesbische Frau wird nicht nur als Opfer der muslimischen Migrant_innen, sondern auch als eine unemanzipierte und unterdrückte Frau konzeptualisiert, die sich weder als Frau noch als Lesbe behaupten könne. Diese und ähnliche Kampagnen erzeugen einen Gegensatz zwischen weiß-europäischer emanzipierter Lesbe vs. muslimisch-unemanzipierter Lesbe, welcher durch eurozentrische Forschungsprojekte stets aktualisiert und bekräftigt wird (z.B. im vom LSVD herausgegebenen Sammelband »Muslime unter dem Regenbogen. Homosexualität, Migration, Islam« 2004 oder im Sammelband »Zwangsverheiratung in Deutschland« in der Forschungsreihe des Deutschen Instituts für Menschenrechte 2008).

Die eurozentrische Wissenschaft interessiert sich kaum dafür, wie Mehrfachausschlussmechanismen Mehrfachdiskriminierungen (re-)produzieren und wie die auf dem Plakat porträtierten Gül und Hassan täglich von ihnen betroffen sind. Wie wir in diesem Text ausführen, werden sie und ihresgleichen von der Wissenschaft als Opfer von Muslim_innen konstruiert. Mit einer Reihe von intransparenten und interessengeleiteten Studien zielt die eurozentrische Wissenschaft gemeinsam mit mehrheitsdeutschen zivilgesellschaftlichen Organisationen auf die Erstellung eines Opfer- und eines Täterprofils von

3 | Vgl. Internetpräsenz des Verbandes: https://www.Isvd.de/ziele/buergerrechte.html. 4 | In einer früheren Kampagne von 2004 hieß es: "Kai ist schwul. Murat auch!", vgl. www.sachsen-anhalt.Isvd.de/?action=archiv\&cat=3\&dok=47. 
Homophobie und Sexismus bzw. der Konstruktion: Schwule vs. Muslime bzw. emanzipierte europäische Lesbe vs. unemanzipierte muslimische Lesbe.

\section{Gerechtigkeitsanspruch des Staates}

Im August 2001 verabschiedete Deutschland das Lebenspartnerschaftsgesetz, auf dessen Basis Lesben und Schwule ihre Partnerschaften nach einem heteronormativen Ehemodell standesamtlich eingehen können. Dieses Gesetz gilt seitdem als Zeichen dafür, dass die BRD einen großen (Fort-)Schritt gegen Homophobie gemacht hat. Der Kampf gegen weitere Formen der Diskriminierung war damit noch nicht gewonnen. Auf Grundlage der EU-Richtlinien und des Amsterdamer Vertrages 1997 wurde Deutschland verpflichtet, ein Gesetz zu verabschieden, das u.a. rassistische, homophobe und sexistische Diskriminierungen verbieten soll (vgl. Çetin 2012). 2006 trat das Allgemeine Gleichbehandlungsgesetz in Kraft, das diese Diskriminierungen in zivilund arbeitsrechtlichen Bereichen beseitigen soll. In diesem Kontext wurden staatliche Gelder für Forschungsprojekte in den Themenbereichen Migration, Integration, Demokratie sowie für den Kampf gegen Extremismus und Terror zur Verfügung gestellt. Mit der Bereitstellung dieser Fördergelder für zivilgesellschaftliche und akademische Projekte begründete der deutsche Staat einen Gerechtigkeitsanspruch, staatliche Universitäten und zivilgesellschaftliche Organisationen begannen, sich mit Themen wie Homophobie, Sexismus, Frauenunterdrückung, Gewalt, Integration sowie Antisemitismus unter muslimischen Migrant_innen zu beschäftigen. Diese staatlich finanzierten Studien wurden zum größten Teil von Organisationen und Wissenschaftler_innen realisiert, die weder von Rassismus noch von Klassendiskriminierung betroffen sind und gehen von einem Widerspruch zwischen homophoben Muslim_innen und homofreundlichen weißen Deutschen aus (vgl. Simon 2008). Die Rolle des Staates in der Verstärkung dieser Konstruktion von Gegensätzen besteht in der ungleichen Verteilung der Ressourcen, die für die antihomophoben »Aufklärungsprojekte« zur Verfügung gestellt werden.

\section{HOMOPHOBIEDISKURSE IN DER WISSENSCHAFT, DER Zivilgesellschaft UNd die Rolle des StaAtes}

Im Folgenden wollen wir das Zusammenwirken von Wissenschaft, Zivilgesellschaft und Staat anhand der Analyse ausgewählter Studien exemplarisch aufzeigen und die damit verbundene (Re-)Produktion des antimuslimischen Rassismus aufdecken (vgl. Çetin 2012). Dafür analysieren wir zwei Studien (MANEO-Umfrage, 2009, und Studie von Simon, 2006) und stellen als 
Gegenmodell die LesMigraS-Studie (2013) vor. Mit ihrer methodischen Vorgehensweise und ihrem intersektionalen Forschungsansatz erachten wir die LesMigraS-Studie als ein Modell für nicht diskriminierende Queer-Studien.

\section{Die MANEO-Umfrage: Gewalterfahrungen von Schwulen und bisexuellen Männern}

Zwischen 2006 und 2008 führte das schwule Anti-Gewalt-Projekt in Berlin MANEO zwei bundesweite Umfragen zum Thema Gewalterfahrungen von Schwulen und bisexuellen Männern in der BRD durch. Auf die erste MANEOUmfrage 2006/2007, bei der ca. 24.000 Personen online einen Fragebogen ausfüllten, folgte $2007 / 2008$ eine zweite Umfrage mit 17.477 Fragebögen (vgl. MANEO 2009: 4). Letztere wurde durch die Stiftung Deutsche Klassenlotterie Berlin finanziert und von Wissenschaftler_innen der Humboldt-Universität, der Evangelischen Hochschule Berlin und dem Wissenschaftszentrum Berlin begleitet (vgl. ebd.: 5). Die zweite Umfrage »steht unter der [...] Fragestellung der Bagatellisierung und möchte aufzeigen, in welchen Bereichen schwulenfeindliche Gewalttaten heruntergespielt bzw. als solche nicht registriert und ernst genommen werden« (ebd.: 10).

Der standardisierte Fragebogen umfasste u.a.

- Fragen zu Gewalterfahrungen [...] und Risikoeinschätzungen sowie

- Fragen zu dem Vorfall, der die Befragten in den letzten 12 Monaten am stärksten betroffen hat,

und wandte sich an in Deutschland lebende bisexuelle Männer und Schwule. Weitere statistische Merkmale wie Alter, Einkommen, sozialer Status oder Wohnort wurden bei der Auswahl der Stichprobe nicht berücksichtigt. Die Umfrage beansprucht keine Repräsentativität (vgl. ebd.: 9).

Wir kritisieren, dass durch die Auswertung der Erhebungen jedoch Opfer- und Täterprofile schwulenfeindlicher Gewalt konstruiert wurden, die in den Medien und öffentlichen Veranstaltungen rassistisch präsentiert wurden (siehe dazu MANEO 2007 und Buntrock 2009).

\section{Konstruktion von Gewaltopfern und -tätern}

Die Teilnehmer der Befragung wurden nach ihren Erfahrungen mit einer oder mehreren von elf juristischen Vorfallformen von Gewalt gefragt. 40,6 Prozent der Befragten (also 7100 Personen) gaben an, in den letzten zwölf Monaten von schwulenfeindlichen Vorfällen betroffen gewesen zu sein (vgl. ebd.: 16). Ohne weitere gesellschaftstheoretische Verankerung des Konzepts Gewalt wurden in der Folge alle der elf juristischen Vorfallformen ohne weitere Differenzie- 
rung als Gewalt zusammengefasst. Im Gegensatz zu dieser fehlenden Differenzierung bei den Gewaltformen macht der Ergebnisbericht genaue Angaben über die Opfer schwulenfeindlicher Gewalt bzw. Hassgewalt:

- 81 Prozent der Befragten seien schwul bzw. homosexuell, die die größte Opfergruppe bilden würden;

- 61 Prozent seien jung (bzw. Schüler an allgemeinbildenden Schulen);

- 92 Prozent seien (weiße) Deutsche.

Die Opfer der Gewalt werden als Gegensatz zu den - angenommenen - Tätern dargestellt. Hierbei ist anzumerken, dass die Einschätzungen zu allen Merkmalen der Täter nach den vorformulierten Antwortoptionen von den Opfern selbst gegeben wurden (vgl. Abou-Chadi/Lippl 2009: 86). Über die Täter_innen der schwulenfeindlichen Gewalt wird festgestellt (vgl. ebd.: 27):

- 86 Prozent der Täter_innen seien männlich;

- 78 Prozent seien jung (18 bis 35 Jahre);

- 40 Prozent hätten einen Migrationshintergrund ${ }^{5}$.

Diese Werte wurden aus den Angaben der Befragten errechnet und sind nicht das Ergebnis rechtskräftiger Verurteilungen. Gewalt im europäischen Kontext wird als ein Phänomen verstanden, an welchem man den Fortschritt oder die Rückständigkeit einer Gesellschaft messen könne. Im Zusammenhang mit dem 11. September, dem Afghanistankrieg oder dem Nahostkonflikt wird Gewalt orientalisiert und als Kontrast zum Europäischen stilisiert (vgl. dazu Said 1978; Attia 2009). Betrachten wir das Zivilisierungskonzept Europas, so sehen wir, dass einige Menschenrechte, z.B. Frauen- und Homosexuellenrechte, als Maßstäbe europäischer Zivilisation festgeschrieben werden und deren Existenz in den als »Orient« imaginierten Ländern in Geschichte und Gegenwart abgesprochen wird.

Die Ergebnisse der MANEO-Umfrage basieren auf einer anonymen Onlinebefragung, die methodisch und sozialwissenschaftlich kritisiert und der

5 | Dem öffentlichen Diskurs folgend sind nicht Migrant_innen aus osteuropäischen oder afrikanischen, asiatischen oder amerikanischen Ländern gemeint; "Migrant_innen “ sind hier Menschen mit Wurzeln in mehrheitlich muslimischen Ländern oder Gebieten - für den deutschen Kontext also v.a. Türk_innen und Kurd_innen als die größten Migrant_innen-Gruppen, oder Araber_innen und Bosnier_innen. Darüber hinaus werden aber auch Menschen in die Schublade "Migration" gesteckt, die etwa als Sinti, Roma oder schwarze Deutsche aufgrund ihrer äußeren Erscheinung als "Migrant_innen" identifiziert werden. Offensichtlich ist es der Blick der weißen deutschen Mehrheitsgesellschaft, der hier entscheidet, über wen gesprochen wird (Wolter/Yılmaz-Günay 2009: 38). 
Manipulation bezichtigt wurde (vgl. Buchterkirchen 2007; Blech 2009). Für die Erhebung des Täterprofils stellte MANEO die per se problematische Zuschreibung »Migrationshintergrund « als eine der möglichen Antwortkategorien zur Auswahl. So zielten die Studie und deren öffentliche Präsentationen darauf ab, junge weiß-deutsche Schwule als Opfer junger, migrantischer und als heterosexuell markierter Männer darzustellen (vgl. MANEO 2009: 19) und die Täter homophober Gewalt zu migrantisieren.

\section{Legitimationsanspruch der Zivilgesellschaft}

In der vorliegenden Studie werden bisexuelle Männer und Schwule als >sexuell anders orientiert <ezeichnet. MANEO erhebt den Anspruch, die ssexuell anders Orientierten gleichberechtigt zu vertreten. Im Fazit der Umfrage wird jedoch deutlich, dass nur sexuell sanders orientierte< Männer und Opfer schwulenfeindlicher Gewalt weiß-deutscher Herkunft vertreten werden.

Für die Selbstbezeichnung werden den Teilnehmern die Optionen »Schwul, Homosexuell, Bisexuell, Heterosexuell, Transsexuell/-gender, Lehne Selbstdefinition ab, Anderes « (ebd.: 12) angeboten. Diese Vorgehensweise ist bedenklich, weil eine wirkliche Selbstbezeichnung nicht mit vorgegebenen Antwortoptionen erfolgen kann. In mehreren Studien wird oft übersehen, dass die befragten Personen sich mit mehreren der vorgegebenen Bezeichnungen identifizieren können. In der MANEO-Umfrage selbst wird nicht Bezug genommen auf die vorgegebenen Identifizierungsmöglichkeiten - vielmehr werden alle Befragten als >sexuell anders orientiert definiert. Die Möglichkeit, sich wirklich selbst zu definieren, wird den Befragten abgesprochen. Die Auftraggeber_innen und Leiter_innen dieser Studie betrachten sexuell anders Orientierte als eine kleine Minderheit in der BRD, die sie in der medialen Öffentlichkeit und in den Jahresberichten durch Diskriminierung und Gewalt seitens junger muslimischer Männer als bedroht darstellen (vgl. MANEO 2009: 17f. und 22ff.).

Die mediale und öffentliche Darstellungspolitik der Studienergebnisse verweist, wie bisher ausgeführt, auf die rassistisch geprägten Interessen der Initiator_innen der Umfrage, die sowohl regierungspolitisch als auch durch die akademische Begleitung unterstützt werden. Als Interessenvertreter_innen einer zur Minderheit gemachten Lesben und Schwulencommunity beanspruchen die Träger_innen der Studien mehr Fördergelder für die Etablierung der Infrastruktur ihrer Lobby- und Beratungsarbeit (vgl. ebd.: 28). Durch die angeblich konstant gebliebenen und/oder zunehmenden Opferzahlen antischwuler Gewalt, die zusätzlich mit wissenschaftlichen Umfragen belegt werden, werden diese Ansprüche gerechtfertigt. 


\section{Simon-Studie zu Einstellungen zur Homosexualität}

Parallel zu den MANEO-Umfragen realisierte Bernd Simon am Institut für Psychologie der Christian-Albrechts-Universität zu Kiel 2006 im Auftrag des LSVD eine Studie über Einstellungen von Jugendlichen mit und ohne >Migrationshintergrund $\mathrm{zu}$ Homosexualität. Das Bundesministerium für Familie, Senioren, Frauen und Jugend sowie die Berliner Senatsverwaltung für Bildung, Jugend und Sport finanzierten die Studie. Ihr Ziel war, homofeindliche Einstellungen von >türkischen, russischen und deutschen< Jugendlichen im Alter zwischen 14 und 20 Jahren zu erforschen und ihre Einstellungen zu Homosexualität miteinander zu vergleichen.

922 standardisierte Fragebögen, beantwortet von Berliner Gymnasiast_innen und Gesamtschüler_innen, wurden in Hinblick auf die Verbreitung von homofeindlichen Einstellungen unter Jugendlichen mit und ohne Migrationshintergrund und auf Ursachen der homofeindlichen Einstellungen ausgewertet.

Sowohl die Verwirklichung einer quantitativen Studie mit vorformulierten geschlossenen Fragen, die die rassistischen Zuschreibungen gegenüber der untersuchten Gruppe mit Migrationshintergrund verstärken, als auch die Auswertung der Fragebögen weisen wissenschaftliche Mängel auf. Studienleiter Bernd Simon geht von der These aus, >dass in den Gruppen mit Migrationshintergrund die Wahrnehmung gruppenbezogener Diskriminierung positiv mit homosexuellenfeindlicher Einstellung zusammenhängt im Sinne einer Konkurrenz der Minderheiten und/oder einer Sündenbockfunktion der homosexuellen Minderheit< (vgl. Simon 2008: 8). Ausgehend von dieser These wird auf die folgenden Forschungsergebnisse abgezielt, die von unterschiedlichen Sozialwissenschaftler_innen und politischen Akteur_innen aus Migrations- und Queerforschung kritisiert wurden (vgl. z.B. Rommelspacher 2007; Yllmaz-Günay 2008):

"1) Jugendliche mit Migrationshintergrund [...] lassen eine homosexuellenfeindlichere Einstellung erkennen als Jugendliche ohne Migrationshintergrund [...].

2) Religiosität und Akzeptanz traditioneller Männlichkeitsnormen sind generell positive Korrelate homosexuellenfeindlicher Einstellung.

3) Persönliche Kontakte zu Homosexuellen sind generell ein negatives Korrelat homosexuellenfeindlicher Einstellung.

4) Der Zusammenhang von Religiosität und homosexuellenfeindlicher Einstellung ist bei Jugendlichen mit türkischem Migrationshintergrund [...] besonders stark ausgeprägt.

5) Diskriminierungswahrnehmungen von Jugendlichen mit Migrationshintergrund [...] sind ein positives Korrelat homosexuellenfeindlicher Einstellung, das Ausmaß der Integration dieser Jugendlichen in die deutsche Gesellschaft ein negatives Korrelat."(Simon 2006: 9) 
Simon stellt bedenkliche und nicht weiter hinterfragte Behauptungen auf: So sei mit der Einführung des Lebenspartnerschaftsgesetzes ein verbessertes Klima für Lesben und Schwule in Deutschland entstanden, es herrsche eine stillschweigende gesellschaftliche Akzeptanz, die jedoch durch bestimmte Angehörige der Migrantengesellschaft bedroht wäre (ebd.: 4f.).

Durch die geografischen, kulturalistischen oder biologistischen Annahmen über die Herkunft der (Groß-)Eltern der Befragten, werden die Jugendlichen nach bestimmten imaginierten Abstammungen konstruiert bzw. kategorisiert und mit einem Migrationshintergrund markiert. Diesen Gruppen werden Jugendlichen ohne Migrationshintergrund gegenübergestellt, die angeblich ein als europäisch konstruiertes Wertesystem vertreten und deutlich weniger homofeindlich seien.

Als türkisch konstruierte Jugendliche werden im nächsten Schritt - ungeachtet ihrer religiösen Zugehörigkeit - als Muslime definiert und der Islam als Ursache für Homosexuellenfeindlichkeit erklärt (ebd.: 24). Eine theoretische Auseinandersetzung mit dem Islam findet nicht statt, andere Religionen werden nicht berücksichtigt.

Mit der letzten Hypothese wird Homosexuellenfeindlichkeit dem Rassismus gegenübergestellt. Dabei werden den als russisch und türkisch konstruierten Jugendlichen Gefühle und Wahrnehmungen rassistischer Diskriminierung zugeschrieben, als wäre diese Art der Diskriminierung kein soziales Phänomen, sondern Resultat individueller Befindlichkeiten. So konstatiert Simon: Je mehr die Befragten sich wegen ihrer Herkunft diskriminiert fühlten, desto homosexuellenfeindlicher seien sie. Er behauptet, dass sich die türkischen und russischen Jugendlichen als Angehörige von Minderheiten in einer Konkurrenz mit homosexuellen Minderheiten sähen und deshalb Homosexuelle negativer bewerteten (vgl. ebd.: 7 f.).

Die Studie überprüft die Ausgangsthesen also nicht mit einem Pre-Test, wie dies den Anforderungen einer wissenschaftlichen Arbeit entspräche, sondern zielt darauf ab, das vom Autor vertretene rassistische Ressentiment zu bestätigen.

Neben diesen kulturalistischen hypothetischen Forschungsergebnissen weist die Simon-Studie methodische und forschungsethische Probleme auf: Unabhängig ihrer Repräsentativität sind die Ergebnisse weder theoretisch noch empirisch begründbar. Das Forschungsteam geht davon aus, dass bestimmte Jugendliche einen Migrationshintergrund haben und kulturell oder religiös eine andere Zugehörigkeit (eine russische und eine muslimische) besitzen. Angesichts dieser Zuschreibungen bzw. Vorannahmen werden den Befragten Selbstbezeichnungen und -definitionen abgesprochen - z.B. sind 88 Prozent der Befragten mit türkischem Migrationshintergrund, die in dieser Studie als am homofeindlichsten klassifiziert werden, seit ihrer Geburt oder frühen Kindheit Teil einer Gesellschaft, die sich als liberal bezeichnet und von sich behauptet, patriarchale Strukturen abgebaut zu haben. 


\section{Die LesMigraS-Studie "Gewalt- und (Mehrfach-)Diskriminierungs- erfahrungen von lesbischen, bisexuellen Frauen und Trans*"}

In diesem Abschnitt wollen wir anhand der qualitativen und quantitativen LesMigraS-Studie die methodischen und politischen Probleme der oben geschilderten Studien zugespitzt aufzeigen und uns gegen identitäre Queerpolitik in der BRD positionieren.

Der Antidiskriminierungs- und Antigewaltbereich der Lesbenberatung Berlin LesMigraS kritisiert in seinen Veröffentlichungen wiederholt die Individualisierung und Kulturalisierung von Homophobie in schwulen und lesbischen Zusammenhängen, in der Öffentlichkeit und in der Politik. Zum Problembereich der Individualisierung beanstandet die Organisation, dass der Fokus der Auseinandersetzung von vielen NGOs sowie von Politik und Öffentlichkeit hauptsächlich auf zwischenmenschliche, körperliche Gewalt im öffentlichen Raum gelegt wird und Aspekte wie strukturelle und institutionelle Diskriminierungen und psychische Gewalt außer Acht gelassen werden. In Bezug auf Kulturalisierung kritisierte LesMigraS, dass in LSBTI*Zusammenhängen das Bild von »weißen, westlichen, emanzipierten LSBTI*« versus »homophoben Migrant_innen« permanent konstruiert und rekonstruiert wird und spricht die Arroganz und Ignoranz der lesbischen, schwulen und Trans*-Zusammenhänge an, mit der diese die Existenz und Präsenz von Schwarzen, von Migrant_innen und People of Color in queeren Kontexten systematisch unsichtbar machen (Saadat-Lendle 2009/2013 und LesMigras 2012a).

In den letzten zehn Jahren hat LesMigraS zahlreiche Studien zu Gewalt und Diskriminierungserfahrungen von queeren Lebensweisen unter die Lupe genommen und aufgezeigt, wie Individualisierung und Kulturalisierung von Homophobie sowie die Ignoranz bezüglich Mehrfachdiskriminierungen in diesen Studien reproduziert werden. LesMigraS betont, dass gerade diese von staatlichen Einrichtungen und von lesbisch-schwulen Organisationen finanzierten Studien dazu benutzt werden, kulturalisierende und individualisierende Haltungen zu rechtfertigen und zu bestärken.

Zwischen 2010 und 2012 realisierte LesMigraS selbst eine sandere Studie, die der Komplexität und Vielfältigkeit der Lebensweisen mit nicht normativen Genderidentitäten und sexuellen Orientierungen gerecht werden sollte. Ziel war es, eine Studie zu verfassen, die

- nicht nur die Normalisierung von Gewaltpraxen gegenüber diesen Menschen verdeutlicht, sondern in der es auch darum ging, zu zeigen, wie unterschiedliche Diskriminierungsformen, z.B. Rassismus, Homophobie, Transphobie, Klassismen, Behinderungs- sowie Altersdiskriminierung zusammenspielen und miteinander verwoben sind; 
- den Interessen, Bedürfnissen und Problemen mehrfachzugehöriger Menschen gleichgeschlechtlicher und transgeschlechtlicher Lebensweise Aufmerksamkeit schenkt;

- die Ressourcen, die Widerständigkeit und das Engagement von (mehrfachzugehörigen) lesbischen, bisexuellen und transgeschlechtlichen Menschen gegen Gewalt und Diskriminierung beachtet und wertschätzt;

- die Thematisierung von Gewalt gegen und Diskriminierung von Trans*Menschen sowie die Entwicklung von Strategien zu deren Bekämpfung explizit erforscht;

- nicht nur Gewalt- sondern auch Diskriminierungserfahrungen in den Vordergrund stellt und diese nicht hauptsächlich auf individualisierte körperliche Gewalt, die im öffentlichen Raum ausgeübt wird, reduziert, sondern psychische und verbale Gewalt ebenso wie Mobbing sowie staatliche Gewalt und Diskriminierung thematisiert;

- sich nicht auf Einstellungsabfragen und auf die Bildung eines Profils über Täter_innengruppen konzentriert und die insbesondere die Konstruktion von polarisierenden und stereotypisierenden Bilder von aufgeklärten Deutschen vs. homophoben Migrant_innen vermeidet (vgl. LesMigraS 2012b).

Um die Komplexität der Lebensrealitäten der geschlechtlichen und sexuellen Vielfalt von lesbischen, bisexuellen und transgeschlechtlichen Menschen $\mathrm{zu}$ erfassen, ermöglichte LesMigraS eine enge Zusammenarbeit zwischen Wissenschaftler_innen, Beratungsstellen und Aktivist_innen und konstruierte einen Fragebogen mit zahlreichen Optionen für Selbstdefinitionen.

LesMigraS strebte damit an, dem klassischen Anliegen der engagierten sozialen Praxis gerecht zu werden, auch die »leisen Stimmen« innerhalb der untersuchten Gruppen zu erfassen. Die enge Zusammenarbeit zwischen Wissenschaftler_innen, Beratungsstellen und Aktivist_innen sollte allen Seiten ermöglichen, ihre spezifischen Kenntnisse, Erfahrungen, Perspektiven und Kompetenzen zusammenzubringen.

Hierbei standen wenig schon in Deutschland bestehende Erfahrungen und Arbeitstraditionen zur Verfügung. Die Studien, die LesMigraS kannte, waren entweder durch NGO's oder durch Wissenschaftler_innen durchgeführt worden, in denen häufig eine Seite die Möglichkeiten und Kompetenzen der anderen genutzt hatte, um seigene Ziele zu erreichen. Gerade bei den Studien im Bereich marginalisierte Menschen ist es aber wichtig, eine enge Zusammenarbeit bei Zielformulierungen, Fragestellungen und Analysen der erworbenen Daten zu ermöglichen, um einer Stigmatisierung, Pauschalisierung und Problematisierung der beforschten Gruppen zu entgehen bzw. problematische sozialwissenschaftliche Forschungen zu sogenannten >Minderheiten $<\mathrm{zu}$ verhindern. 
Diese Zusammenarbeit ist, auch wenn sie aus der Sicht von LesMigraS nicht vollständig zufriedenstellend und reibungslos verlief, die Stärke dieser Studie. LesMigraS führte die Forschung zu Gewalt und Mehrfachdiskriminierungserfahrungen von lesbischen, bisexuellen Frauen und Trans* gemeinsam mit Wissenschaftler_innen durch, alle Forscher_innen verfügten über jeweils unterschiedliche Verantwortungsbereiche, Zuständigkeiten und Perspektiven.

Für die Konstruktion eines offeneren Fragebogens stellte LesMigraS zu Beginn der Studie fest, dass »die im akademischen Feld so differenziert und intensiv geführten Debatten zu >Queer<, >Intersektionalität< oder auch zur >Dekonstruktion von Identitäten vereinbar sind « (Castro Varela 2012: 10). LesMigraS war sich im Vorfeld bewusst, dass quantitative Erhebungen schließlich nur funktionieren, wenn »Kategorien festgelegt werden können und Erfahrungen berechenbar sind. Solche Kategorien benennt LesMigraS allerdings selbst als Grund und Effekt von Diskriminierungspraxen.« (Ebd.: 11)

Eine Infragestellung der Kategorien schien, zumindest vor dem Hintergrund der LesMigraS damals verfügbaren Erfahrungen, mit quantitativen Erhebungen kaum möglich zu sein. Um diesem Problem zu entgehen, sollte die Studie den Teilnehmenden im Fragebogen viele Wahlmöglichkeiten geben, um sich selbst zu bestimmen. So wurde beim Aufbau der Studie darauf geachtet, dass die potenziellen Studienteilnehmer_innen möglichst wenig Vorannahmen, Zuschreibungen und Ausgrenzungen ausgesetzt waren. Maria do Mar Castro Varela, wissenschaftliche Leiterin der Studie, stellte diesbezüglich fest:

"Das Dilemma, mit welchem ein Umgang gefunden werden musste, wäre zu umschreiben als 'Komplexitätı versus `Präzision`. Genauer betrachtet zeigt sich letztendlich natürlich, dass die Unschärfe, mit der LesMigraS bei der Auswertung zu kämpfen hatte, ein kleiner Preis ist gegenüber der ständigen Wiederholung von Stereotypen, die aus den oft kruden Simplifizierungen anderer Erhebungen resultieren. [...] In der Darstellung der Ergebnisse wurde deshalb Wert darauf gelegt, die Unschärfe und Widersprüche nicht zu glätten, sondern sie herauszuarbeiten, weil sie mehr über die Realität von Diskriminierung aussagen als die scheinbaren 'klaren، Ergebnisse, die immer wieder die Differenzen zwischen der 'Mehrheitı und den 'Anderen ‘ festzurren.“ (Ebd.: 12)

Die in diesem Sinne offen gehaltenen Fragen hatten ebenso den »sehr positiven Effekt, dass gezeigt werden konnte, dass starre Kategorisierungen von einer Vielzahl der Befragten abgelehnt werden. Ein Großteil der angebotenen Kategorien ist kritisch hinterfragt worden. Die Teilnehmenden nutzten so bei der Beschreibung ihrer >Identität< sehr kreativ jede Frage, die die Möglichkeit zur Selbstbeschreibung bzw. Selbstdefinition bot, um Kategorien zu hinterfragen und zu irritieren.« (LesMigraS 2012b: 205) 
Im Gegensatz zu anderen Studien, die von einer klaren Selbstrepräsentation ausgehen, deutet so die LesMigraS-Studie auf die Brüchigkeit von Identitätskategorien hin und eröffnet zudem Räume, um diese sichtbar werden zu lassen. So wurden auf die Frage nach den Selbstbezeichnungen bezüglich >nationaler/ethnischer/kultureller< Zugehörigkeit vielfältige und differenzierte Antworten gegeben, während etwa die Bezeichnung »Mensch mit Migrationshintergrund « Kritik erfuhr. In Bezug auf die Kategorie Migration gab eine Teilnehmende an: »Ich habe zwar einen Migrationshintergrund, aber es klingt wie ne Krankheit. Ich bin deutsch.« Eine andere beschrieb sich als »deutsch, migrantisiert«. In Bezug auf die Kategorien Gender, Geschlecht und sexuelle Lebensweise gaben die Befragten u.a. an: »Ich definiere mich je nachdem, welcher Sex-Gender-Anteil grad im Vordergrund ist«; »Ich entdecke mich gerne jeden Tag neu, definiere mich aber politisch als Frau«; »lesbisch + trans liebend«; »alles, aber nicht heterosexuell«; »meistens männlich; manchmal einfach planlos«; »multidimensional weiblich«, »lesbischlesbisch-poly-queer im Fluss«. Weit über ein Drittel (ca. 40\%) der Teilnehmenden an der Studie von LesMigraS gab an, erlebt zu haben, dass z.B. im Bildungsbereich diskriminierende Sprüche gegenüber von Lesben, Bisexuellen und Trans* Schüler_innen vom Lehrpersonal ignoriert oder selbst geäußert wurden. Über zwei Drittel (ca. $73 \%$ ) erlebten, dass ihre Leistungen im Bildungsbereich aufgrund ihrer nonkonformen sexuellen und geschlechtlichen Lebensweise vergleichsweise schlechter bewertet wurden. Auch im Bereich Arbeit erlebte mehr als ein Viertel der Teilnehmenden (27,9\%) - bei Trans*Personen jeder zweite $(50 \%)$ - »sehr oft« und »eher oft« Diskriminierungen, weit über ein Viertel (30,7\%) machte Mobbingerfahrungen, mindestens ein Fünftel $(20,6 \%)$ erlebte, dass sie ihre_n Partner_in besser nicht mit zu einer betrieblichen Veranstaltung mitbringen sollten. Im Bereich Polizei und Justiz meinte ebenso über ein Viertel der Teilnehmenden (26,8\%), dass eine lesbische/bisexuelle Lebensweise negative Auswirkungen auf die Chancen hat, von der Polizei geschützt zu werden. Lesbische, bisexuelle Frauen und Trans* (LBT) mit Migrationsbiografie wurden am Arbeitsplatz mindestens 10 Prozent mehr diskriminiert (37,5\%) als nicht mehrfachdiskriminierte weiße deutsche LBT. Sie gaben zu 10 Prozent häufiger an $(30,4 \%)$, dass ihnen mindestens einmal nahegelegt wurde, ihre_n Partner_in nicht $\mathrm{zu}$ einer betrieblichen Veranstaltung mitzubringen. Im öffentlichen Raum erlebten sie 28 Prozent mehr Diskriminierungen und Gewalt, im Gesundheitsbereich, bei Ämtern und Behörden 22 Prozent häufiger Diskriminierung, und sie äußerten doppelt so oft wie nicht mehrfachdiskriminierte weiße deutsche LBT (7\%) das Gefühl, dass ihre Mehrfachzugehörigkeit negative Auswirkungen auf die Chancen habe, von der Polizei geschützt zu werden. 


\section{Vergleich der Studien}

Es ist an die Zeit, dass Wissenschaftler_innen Methoden weiterentwickeln und anwenden, die die Untersuchung von Mehrfachdiskriminierungen und die Infragestellung von Kategorien ermöglichen. Nur so kann es gelingen, die Komplexität der Lebensrealitäten und der geschlechtlichen und sexuellen Vielfalt zu erfassen.

Wie oben ausgeführt, werden die Gewalterfahrungen von lesbisch-schwulen Menschen in den meisten Studien individualisiert. Sie beziehen sich häufig auf individuelle Diskriminierungserfahrungen von weißen Schwulen, selten auch Lesben, durch eine andere Person, angeblich oft Migrant_innen. So wird ein Bild von einer aufgeklärten deutschen Gesellschaft konstruiert, die durch Migrant_innen bedroht wird. Formen struktureller Gewalt und Diskriminierung, damit meinen wir Diskriminierung und Gewalt durch Gesetze, Schule, Medizin, Justiz, Polizei, ..., werden in den Studien (z.B. MANEO, 2oo9; Simon, 2006) nicht erwähnt. Ebenso wenig wird beachtet, inwiefern Menschen, die von Mehrfachdiskriminierungen betroffen sind, weit über diese Diskriminierungspotenziale hinaus Diskriminierung und Gewalt erleben. Würden diese strukturellen Formen berücksichtigt, dann ergebe sich durchaus ein anderes Bild von Deutschland, welches nicht zum polarisierenden Bild der fortschrittlichen Deutschen im Kontrast zu den rückschrittlichen Migrant_innen passt.

Wenn Studien, wie die vom LSVD oder MANEO, Diskriminierungserfahrungen von Lesben und Schwulen mit einer Migrationsbiografie thematisieren, so stellen sie diese meist als Opfer patriarchaler familiärer, religiöser und/ oder kultureller Strukturen, nicht aber struktureller Diskriminierung dar. Die Studie von LesMigraS zeigt allerdings, dass Diskriminierungserfahrungen in der Familie von LBT mit Migrationsbiografien nur um 6 Prozent häufiger angegeben werden als von nicht mehrfachdiskriminierten weißen deutschen LBT (48\% vs. $42 \%)$.

Da die Thematisierung von Homophobie und Trans*Diskriminierung im Bereich Bildung, Arbeit, Behörden, Ämter, Dienstleistungen - d.h. der große Bereich struktureller und institutioneller Diskriminierung und Gewalt - nicht zu dem Bild einer grundsätzlich aufgeklärten deutschen Gesellschaft passt, das der LSVD, MANEO und andere ähnliche Studien vermitteln, werden solche Fragen weder gestellt noch beforscht. Dies scheint uns jedoch fatal, trägt doch eine solchermaßen strukturelle Gewalt mit dazu bei, dass individuelle Gewaltformen entstehen können, ausgeführt von Menschen mit oder ohne Migrationsbiografie, die in dieser Gesellschaft aufwachsen und von ihr geprägt werden. 


\section{FAZIT}

Anhand der ausgewählten Studien, die sich dem Thema Homophobie in der BRD widmen, zeigten wir, dass die medialen, wissenschaftlichen und politischen Diskussionen über Homophobie in den 200oer Jahren in einer Kooperation zwischen Staat, Wissenschaft, Zivilgesellschaft und Medien kontinuierlich durchgeführt werden. Der Staat als Geldgeber, die Wissenschaft als Legitimationsinstrument, die Zivilgesellschaft als Auftraggeberin und die Medien wirken bei der Verbreitung und Verfestigung eines antimuslimischen Homophobiediskurses erfolgreich zusammen.

Sowohl durch die Analyse der quantitativen Studien als auch mittels unserer eigenen wissenschaftlichen Auseinandersetzungen mit mehrdimensionalen Diskriminierungen stellen wir fest, dass in zivilgesellschaftlichem wie auch in wissenschaftlichem Kontext eine Bedeutungsverschiebung der Homophobie stattgefunden hat. Eine Mehrzahl der Studien fokussiert ausschließlich auf eine Homophobie, der schwule oder bisexuelle Männer ausgesetzt sind und die nicht von weiß deutscher Mehrheitsgesellschaft ausgeht. Die Diskriminierungserfahrungen von Lesben und bisexuellen Frauen werden in vielen Studien entweder nur am Rande behandelt oder gar nicht beachtet. Die Diskriminierungserfahrungen von allen potenziellen Betroffenen der Homophobie werden leider oft homogenisiert. Dabei werden klassen- und schichtspezifische Aspekte, gender- und geschlechterspezifische Besonderheiten und durch Rassismus bedingte Diskriminierungen, die sich (in-)direkt mit Homophobie überschneiden, häufig außer Acht gelassen. Die Studien präsentieren und (re-) produzieren stetig das Profil eines Opfer von Homophobie, das weiß, mittelschichtig und schwul ist, während diejenigen als homophob bzw. schwulenfeindlich dargestellt werden, die weder als schwul noch als weiß gelten und als Angehörige eines bildungsfernen Unterschichtmilieus klassifiziert werden.

Sowohl die analysierten Studien als auch zahlreiche Anti-Homophobiekampagnen in der BRD konstruieren Homophobie als eine Eigenschaft von jungen, muslimischen und/oder russischen bildungsfernen Männern, denen gleichzeitig Merkmale wie Gewaltbereitschaft oder Kriminalität zugeschrieben werden.

Homophobie wird in diesen pseudowissenschaftlichen und politischen Projekten rassifiziert, kulturalisiert und klassifiziert. Die bundesrepublikanische Gesellschaft wird in diesem Homophobiediskurs nicht nur als »homophobe Muslim_innen vs. homophile Deutsche« polarisiert, auch die in der BRD lebenden Muslim_innen werden gegeneinander gestellt. Queere Muslim_innen werden in diesen antihomophoben Projekten als Opfer des Islam bzw. der muslimischen Familien oder Gesellschaft repräsentiert.

Auf dem ersten Wissenschaftskongress der BMH haben wir uns als Queer of Color positioniert und die Öffentlichkeit auf die ausgrenzenden Ungleich- 
heitsverhältnisse in der deutschen Queerpolitik und -studien aufmerksam gemacht. Am Beispiel des Kongresses haben wir auch angemerkt, dass die Auswahl der queeren Referent_innen nicht frei von diesen Ungleichheitsverhältnissen war. Die Mitarbeiter_innen von LesMigraS waren bspw. nicht nur NGO-Mitarbeiter_innen, sondern gleichzeitig wissenschaftliche Mitarbeiter innen ihrer eigenen Studie. Diese wertvolle Erfahrung wurde auf diesem Kongress nicht anerkannt, die Studie durfte nicht vorgestellt werden. Grund war, dass das Kongressvorbereitungsteam der BMH die Qualifikationen von Saideh Saadat-Lendle, Leiterin von LesMigraS und Begleiterin der LesMigraS-Studie »Gewalt und (Mehrfach-)Diskriminierungen von lesbischen, bisexuellen Frauen und Trans ${ }^{\star} \ll$, als nicht wissenschaftlich eingestuft hat. Da sie nicht promoviert ist bzw. zurzeit keine wissenschaftliche Tätigkeit in einem Forschungsinstitut oder an einer Hochschule ausübt, sei sie zur Vorstellung der Studie nicht geeignet. So wurde eine wertvolle Erfahrung im Bereich partizipativer Forschung zu Lebensrealitäten marginalisierter Gruppen abgewertet, anstatt diese aufzugreifen, wertzuschätzen und zu vertiefen. Auch die anderen Referent_innen of Color, z.B. Maria Virginia Gonzalez Romero von Via Bayern e.V. Verband für interkulturelle Arbeit und Jacek Marjański von Rubicon Köln erhielten auf dem Kongress nicht ausreichend Zeit, ihre eigenen wissenschaftlichen und sozialarbeiterischen Erfahrungen bezüglich Mehrfachdiskriminierungen ihrer Klient_innen zu referieren. Ihre Qualifikationen wurden auf dem Kongress der BMH aus denselben Gründen wie bei Saideh Saadat-Lendle nicht anerkannt. Da sich ein ganzer Block dieses Kongresses mit der »Partizipativen Forschung « auseinandergesetzt hat, bleibt uns nichts anders übrig als zu hoffen, dass die Reflexion über unsere Kritik in Bezug auf Auswahl der Referent_innen des Kongresses die zukünftige Praxis der wissenschaftlichen Kongresse, darunter auch von der BMH, zugunsten eines partizipativen Ansatzes beeinflussen kann.

In diesem Beitrag haben wir dargelegt, dass Queerstudies und eine adäquate Queerpolitik ohne eine Auseinandersetzung mit Rassismen und klassenspezifischen sozialen Diskriminierungen nicht möglich sind. Unsere bundesweiten und lokalen Studien und Projekte, welche die Lebensperspektiven von Queer of Color in Deutschland sichtbar machen und ein Zeichen gegen diskriminierende eurozentrische Dominanzverhältnisse setzen, haben wir als Alternative für die Wissensproduktion hervorgehoben. Die weißen queeren Wissenschaftler_innen und Aktivist_innen fordern wir auf, sich mit mehrdimensionalen Diskriminierungen auseinanderzusetzen, indem sie mit Queer of Color und nicht für oder über Queer of Color sprechen. 


\section{LITERATUR}

Abou-Chadi, Tarik/Lippl, Bodo (2009): Materialband zu den Ergebnissen der MANEO-Umfrage 2 (2007/2008) Gewalterfahrungen von schwulen und bisexuellen Jugendlichen und Männern in Deutschland, www.maneo-tole ranzkampagne.de/pdf/maneo-umfrage2-materialband.pdf, zuletzt aufgerufen am 04.05.2014.

Attia, Iman (2009): Die »westliche Kultur« und ihr Anderes. Zur Dekonstruktion von Orientalismus und antimuslimischem Rassismus, Bielefeld: transcript.

Blech, Norbert (2009): MANEO-Umfrage gezielt manipuliert?, www.queer.de/ detail.php?article_id=10906, zuletzt aufgerufen am 03.01.2014.

Buchterkirchen, Ralf (2007): MANEO: Opfertelefon auf Feindbildsuche, http:// verqueert.de/opfertelefon-auf-feindbildsuche/, zuletzt aufgerufen am 03.01. 2014 .

Bundesministerium für Familie, Senioren, Frauen und Jugend (2008): Zwangsverheiratung in Deutschland, www.bmfsfj.de/RedaktionBMFSFJ/Broschue renstelle/Pdf-Anlagen/Zwangsverheiratung-_2oForschungsreihe-Band_2 o1,property=pdf,bereich=bmfsfj, sprache=de,rwb=true.pdf, zuletzt aufgerufen am 04.05.2014.

Buntrock, Tanja (2009): Angst im schwulen Kiez, www.tagesspiegel.de/berlin/ polizei-justiz/berlin-schoeneberg-angst-im-schwulen-kiez/1428078.html, zuletzt aufgerufen am 05.04.2014.

Castro Varela, Maria do Mar (2012): Traurige Forschung, www.lesbenbera tung-berlin.de/tl_files/lesbenberatung-berlin/Gewalt\%20\%28Dokus,Auf saetze...\%29/Dokumentation\%20Studie\%2oweb_sicher.pdf, zuletzt aufgerufen am 07.05.2014.

Çetin, Zülfukar (2012): Homophobie und Islamophobie. Intersektionale Diskriminierungen am Beispiel binationaler schwuler Paare in Berlin, Bielefeld: transcript.

LesMigraS (undatiert), Was ist Mehrfachdiskriminierung?, www.lesmigras. de/mehrfachdiskriminierung.html, zuletzt aufgerufen am 07.05.2014.

LesMigraS (2012a): Unser Gewaltverständnis, www.lesmigras.de/tl_files/lesmi gras/Texte/2012_aug_gewaltverstaendnis.pdf, zuletzt aufgerufen am 07.04. 2014 .

LesMigraS (2012b): »... nicht so greifbar, und doch real ...«. Eine quantitative und qualitative Studie zu Gewalt und (Mehrfach-)Diskriminierungserfahrungen von lesbischen, bisexuellen Frauen und trans* in Deutschland, www.lesmigras.de/tl_files/lesbenberatung-berlin/Gewalt\%20\%28Dokus, Aufsaetze...\%29/Dokumentation\%2oStudie\%2oweb_sicher.pdf, zuletzt aufgerufen am 10.02.2014. 
LSVD Berlin-Brandenburg (Hg.) (2004): Muslime unter dem Regenbogen. Homosexualität, Migration und Islam, Berlin: Querverlag.

LSVD (2007): Kai ist schwul. Murat auch!, www.sachsen-anhalt.lsvd.de/?action $=$ archiv\&cat=3\&dok=47, zuletzt aufgerufen am 24.01.2014.

LSVD (2012): Gül ist lesbisch - Hassan ist schwul. Neue Plakatkampagne des BÜNDNISSES GEGEN HOMOPHOBIE, https://berlin.lsvd.de/neuigkeiten/ neue-plakatkampagne-startet/, zuletzt aufgerufen am 05.04.2014.

MANEO (2009): Gewalterfahrungen von schwulen und bisexuellen Jugendlichen und Männern in Deutschland. Ergebnisse der MANEO-Umfrage 2 (2007/2008), www.maneo-toleranzkampagne.de/pdf/maneo-umfrage2bericht.pdf, zuletzt aufgerufen am 03.01.2014.

Rommelspacher, Birgit (2006): Interdependenzen- Geschlecht, Klasse und Ethnizität. Beitrag zum virtuellen Seminar Mai 2006, www.birgit-rommelspacher.de/intedependenzen.pdf, zuletzt aufgerufen am 05.04.2014.

Rommelspacher, Birgit (2007): Stellungnahme zur Untersuchung von Prof. Bernd Simon: Einstellungen zu Homosexualität: Ausprägungen und sozialpsychologische Korrelate bei Jugendlichen mit und ohne Migrationshintergrund. Unveröffentlichtes Manuskript.

Saadat-Lendle, Saideh (2009): Mehrfachzugehörige als Impulsgeberinnen, www.lesmigras.de/tl_files/lesmigras/Fachtag\%2010\%20Jahre\%2oLesMigraS/grusswort_saadat-lendle.pdf, zuletzt aufgerufen am 07.04.2014.

Saadat-Lendle, Saideh (2013): Kategorien? Nein danke! Saideh Saadat-Lendle über die Arbeit von LesMigraS in Berlin, www.vielfalt-statt-gewalt.de/file admin/vielfalt-statt-gewalt/pdf/Broschuere_AGG_2013.pdf, zuletzt aufgerufen am 07.04.2014.

Said, Edward (1978): Orientalism, London: Random House.

Simon, Bernd (2008): „Einstellungen zur Homosexualität: Ausprägungen und psychologische Korrelate bei Jugendlichen ohne und mit Migrationshintergrund (ehemalige UdSSR und Türkei)«, in: Zeitschrift für Entwicklungspsychologie und Pädagogische Psychologie 40 (2), S. 87-99.

Wolter, Salih/Y1lmaz-Günay, Koray (2009): »>Muslimische< Jugendliche und Homophobie - braucht es eine zielgruppenspezifische Pädagogik?«, in: Stephan Bundschuh/Birgit Jagusch/Hanna Mai (Hg.), Facebook, Fun und Ramadan. Lebenswelten muslimischer Jugendlicher, Düsseldorf, S. 34-38. Y1lmaz-Günay, Koray (2008): Ethno oder lieber Homo? Kreuzberger Nächte sind lang, www.zag-berlin.de/antirassismus/archiv/53kreuzberger.html, zuletzt aufgerufen am 10.02.2014. 